\title{
Otolaryngologic Manifestation and Long-Term Outcome in Mild COVID-19: Experience from a Tertiary Care Centre in India
}

\author{
Smriti Panda $^{1} \cdot$ Adil Mohamed $^{1} \cdot$ Kapil Sikka $^{1} \cdot$ Anupam Kanodia $^{1} \cdot$ \\ Pirabu Sakthivel $^{1}$ - Alok Thakar ${ }^{1} \cdot$ Sushma Bhatnagar $^{2} \cdot$ Anant Mohan $^{3}$. \\ Ved Prakash Meena ${ }^{4} \cdot$ Pavan Tiwari $^{3} \cdot$ Biswajeet Sahoo $^{5} \cdot$ Lalit Dar $^{5}$. \\ Saurabh Vig $^{2} \cdot$ Rohit Garg ${ }^{4} \cdot$ Chitresh Kumar ${ }^{6}$
}

Received: 1 September 2020/Accepted: 7 October 2020/Published online: 14 October 2020

(C) Association of Otolaryngologists of India 2020

\begin{abstract}
This study endeavours to comprehensively the study the spectrum of ENT manifestations in mild and asymptomatic COVID-19 and observe the natural course of anosmia and dysgeusia consequent to SARS-Cov-2 infection. A prospective cohort study was undertaken at a tertiary care centre in India on admitted patients with RT-PCR proven COVID-19. Patients were included provided the baseline National Early Warning Score (NEWS) was less than 4. Patients were screened for ENT manifestations using a questionnaire at baseline, 7 days, 14 days and 28 days. 225 patients were included in the study. Of these complete data at 4 weeks was available for 210. Out of 145 patients with mild COVID-19 (asymptomatic $=80$ ), ENT manifestations accounted for $66.2 \%$ of all symptoms. Smell and taste disturbance had an overall incidence of $20 \%$ and $45 \%$ of ENT manifestations. Temporal trends of the recovery rate of anosmia and dysgeusia were $53.6 \%$
\end{abstract}

Kapil Sikka

kapil_sikka@yahoo.com

1 Department of Otorhinolaryngology and Head and Neck Surgery, Teaching Block 4th Floor, All India Institute of Medical Sciences, New Delhi, Ansar Nagar East, New Delhi 110029, India

2 Department of Oncoanesthesia, All India Institute of Medical Sciences, New Delhi, New Delhi, India

3 Department of Pulmonary Medicine, All India Institute of Medical Sciences, New Delhi, New Delhi, India

4 Department of Medicine, All India Institute of Medical Sciences, New Delhi, New Delhi, India

5 Department of Microbiology, All India Institute of Medical Sciences, New Delhi, New Delhi, India

6 Department of Surgical Oncology, All India Institute of Medical Sciences, New Delhi, New Delhi, India and $66.7 \%$ at 2 weeks, respectively. Corresponding rates at 3 weeks were $89.29 \%$ and $86.7 \%$, respectively. By the end of 4 weeks $96 \%$ of the patients had recovered completely. The incidence of anosmia and dysgeusia in this study parallels the rates reported from other Asian countries, albeit, lower than the rates quoted from the Western Hemisphere. We recommend structured reporting of all ENT manifestations especially smell and taste disturbances to accurately identify individuals infected with SARSCov-2.

Keywords COVID-19 · SARS-Cov-2 - Anosmia ·

Dysgeusia $\cdot$ ENT $\cdot$ Otorhinolaryngology

\section{Introduction}

The COVID-19 pandemic which began in December 2019 at the Hubei province in China has amounted to 21 million cases and claimed 7 lakh lives worldwide at the time of writing this manuscript [1]. The Indian subcontinent currently accounts for 6 lakh active cases and a total of 50,000 deaths. There has been a surge in the scientific literature related to the natural course of this novel disease process. It is now understood that $50 \%$ of the individuals who test positive on SARS-Cov-2 RT-PCR are asymptomatic and almost $87 \%$ have mild disease (mild clinical symptoms and no abnormal findings on chest imaging) [2,3]. This particular subset of patients has epidemiological significance in the transmission of the disease process in the community. Otolaryngologic symptoms comprise a large gamut of the mild COVID-19 symptomatology with reports of smell and taste disturbance being the only manifestation in "asymptomatic" individuals [4]. The aim of the present study, therefore, was to analyze the spectrum of 
otolaryngologic manifestations in a homogenous subset of mild and asymptomatic COVID-19 patients and also to gain insight into the long-term recovery rates of smell and taste disturbances. Understanding these early-onset signs and symptoms would have immense implication towards the existing testing strategy and isolation.

\section{Materials and Methods}

This was a prospective cohort study conducted at a designated COVID-19 facility (NCI-A.I.I.M.S, Jhajjar). The authors assert that all procedures contributing to this work comply with the ethical standards of the institutional guidelines of All India Institute of Medical Sciences, New Delhi on human experimentation and with the Helsinki Declaration of 1975, as revised in 2008. All consecutive patients with SARS-Cov-2 RT-PCR positive admitted from 23-04-2020 to 29-06-2020 were included. Patients who had a NEWS (National Early Warning Score) of less than or equal to 4 or in other words mild disease at admission were included in this study. Patients were interviewed at admission to obtain information related to demography, presence and duration of symptoms as depicted in Fig. 1. Information was also obtained related to comorbid illnesses if any. Patients were longitudinally followed up by telephonic interview at 7 days, 14 days and 1 month.

All statistical analysis was performed using MedCalc version 19.4.1. For univariate analysis using a categorical variable, Fischer's Exact Test was used. For continuous variables, the distribution was found to be non-normal and therefore, Mann-Whitney $U$ test was performed. A $p$ value of less than 0.05 was taken as statistically significant. All confidence intervals mentioned are $95 \%$ confidence intervals.

\section{Results and Analysis}

225 patients fulfilled the inclusion criteria and were included in this study. The mean age of the cohort was $34.96 \pm 13.4$ years (range: 5-65 years). At baseline, 80 (35.5\%) were found to be asymptomatic. The remaining 145 patients were mildly symptomatic with NEWS less than 4 as shown in Table 1.30 patients $(13.3 \%)$ were found to have comorbid illnesses with diabetes and hypertension being the most frequently encountered. Table 1 summarises the salient demographic features of this cohort.

\section{Distribution of Symptoms at Baseline}

Graphical representation of symptoms at baseline is depicted in Fig. 1. Out of the 145 patients with mild disease at the outset, otolaryngologic symptoms could be identified in $96(66.2 \%)$. Of the non-ENT symptoms, fever and constitutional symptoms were the most common ( $n=104$ and 108, respectively) followed by cough

Distribution of Symtoms at Baseline

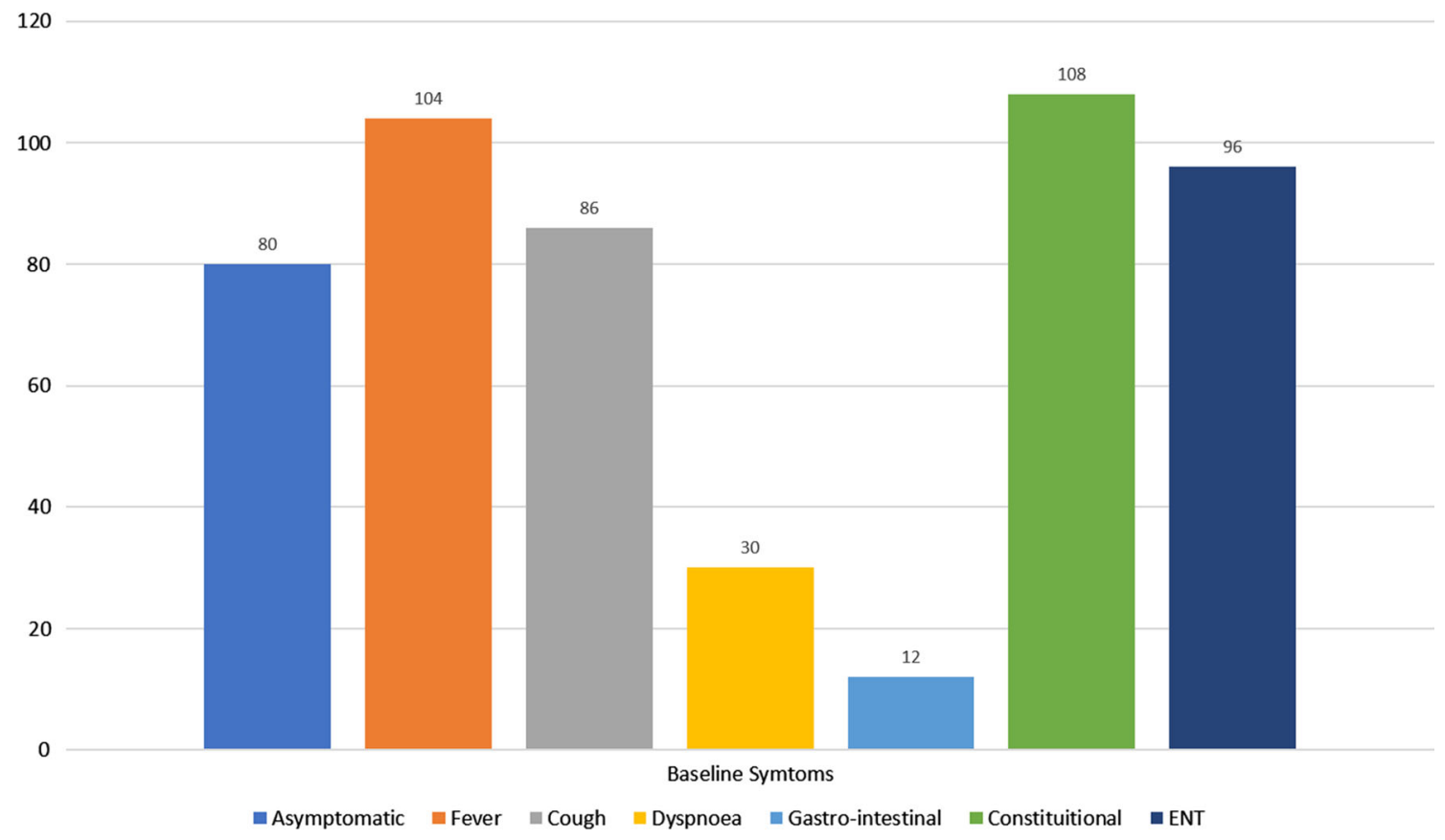

Fig. 1 Distribution of symptoms at baseline 
Table 1 Salient baseline characteristics of the study cohort

\begin{tabular}{ll}
\hline $\mathrm{n}$ & 225 \\
Male:female & $159: 63$ \\
Transgender & 3 \\
Age (mean \pm SD) & $34.96 \pm 13.4$ (range 5-65) \\
Symptomatic & 145 \\
Asymptomatic & 80 \\
NEWS-2 Score & \\
0 & 195 \\
1 & 29 \\
2 & 1 \\
Comorbidities & \\
Diabetes & 13 \\
Hypertension & 8 \\
Bronchial asthma/COPD & 2 \\
Pulmonary tuberculosis & 1 \\
Malignancy & 1 \\
Hypothyroidism & 2 \\
Chronic kidney disease & 2 \\
Seizure disorder & 1 \\
Immunosuppression & 0 \\
\hline
\end{tabular}

$(n=86)$ and shortness of breath $(n=30)$. A gastrointestinal manifestation was noted in only $8.27 \%$.

\section{Distribution of ENT Manifestations at Baseline}

Graphical representation of ENT manifestation is depicted in Fig. 2. Out of the 96 patients with ENT symptoms are baseline, the most common symptom was odynophagia $(n=61,63.5 \%)$, followed by smell and taste disturbance.

In the entire cohort of 225 patients, 29 (12.8\%) had sudden onset anosmia and $39(17.33 \%)$ had dysgeusia. Combined smell and taste disturbance were noted in 21 (9.3\%) patients. Nasal obstruction as a cause of anosmia was ruled out as the former was noted in only 3 patients. None of the patients has anosmia or dysgeusia as their only baseline symptom. No significant difference in terms of the temporal association was noted in the onset pattern of smell and taste disturbance with other baseline symptoms. However, in 14 patients, smell and taste disturbance preceded the onset of other symptoms.

Minor ENT manifestations which were reported by a small number of patients included dizziness, epistaxis, sneezing and aphthous ulcers.

\section{Recovery Pattern of Sudden Onset Anosmia and Dysgeusia}

Follow-up data at 4 weeks was available for 210 patients. Of the 15 patients lost to follow-up, 7 patients had anosmia/dysgeusia. Of the 210 patients with complete follow-up data, mean duration of anosmia and dysgeusia was 8.10 days (range: 1-29 days) and 6.53 days (range: 1-29 days), respectively. Figure 3 depicts the recovery pattern of anosmia and dysgeusia in this study. By 2 weeks, $53.6 \%$ of the patients with anosmia and $66.7 \%$ of the patients with dysgeusia had a complete recovery. This increased to $89.29 \%$ in case of anosmia and $86.7 \%$ in the case of dysgeusia at the end of 3 weeks. By the end of 4 weeks, $96 \%$ of the patients had recovered completely.

\section{Univariate Analysis}

The impact of age, sex, comorbidity, presence of other symptoms and NEWS on the incidence of anosmia/dysgeusia was studied using a univariate model as shown in Table 2 . The only statistically significant correlation was seen in patients with comorbid illnesses. Patients with comorbidities were 2.28 times more likely to present with anosmia or dysgeusia.

\section{Discussion}

In this prospective cohort study conducted on 225 patients with mild COVID-19, at least one ENT manifestation could be identified in $62.2 \%$ of the patients. The most common otolaryngologic symptom was odynophagia $(63.5 \%)$ followed by smell and taste abnormality $(n=45$, overall-20\%, $46.8 \%$ of all ENT symptoms). Pharyngeal symptoms were found to be more commonly associated with COVID-19 than nasal symptoms like obstruction and discharge in studies from Italy and Egypt $[5,6]$. The smell and taste disturbance was found to be temporary with $96 \%$ of the patients regaining their function by 4 weeks. No significant association could be demonstrated with disease severity within the mild cohort, nor could we identify any correlation with sex or age. However, patients with comorbidities $(n=30,13.3 \%)$ were more likely to present with either smell or taste disturbance (RR 2.28, $p$ 0.04, 95\% CI 2.5-16.3).

Mao et al. in their multi-institutional study on 214 patients were the first to highlight the neurological affectations consequent to COVID-19 [7]. Their series reported $5.1 \%$ incidence of anosmia and $16.8 \%$ incidence of dizziness. As opposed to the $20 \%$ incidence identified in our study. The study by Mao et al. however, had an admixture of patients with varying disease severity unlike our homogenous population of NEWS ranging between 0 and 2. Subsequently, there have been several reports focusing on the anosmia and dysgeusia. European studies demonstrated a much higher incidence, ranging from $56.9 \%$ in the study by Mercante et al. (I-SNOT 22 on 204 patients) to 
70

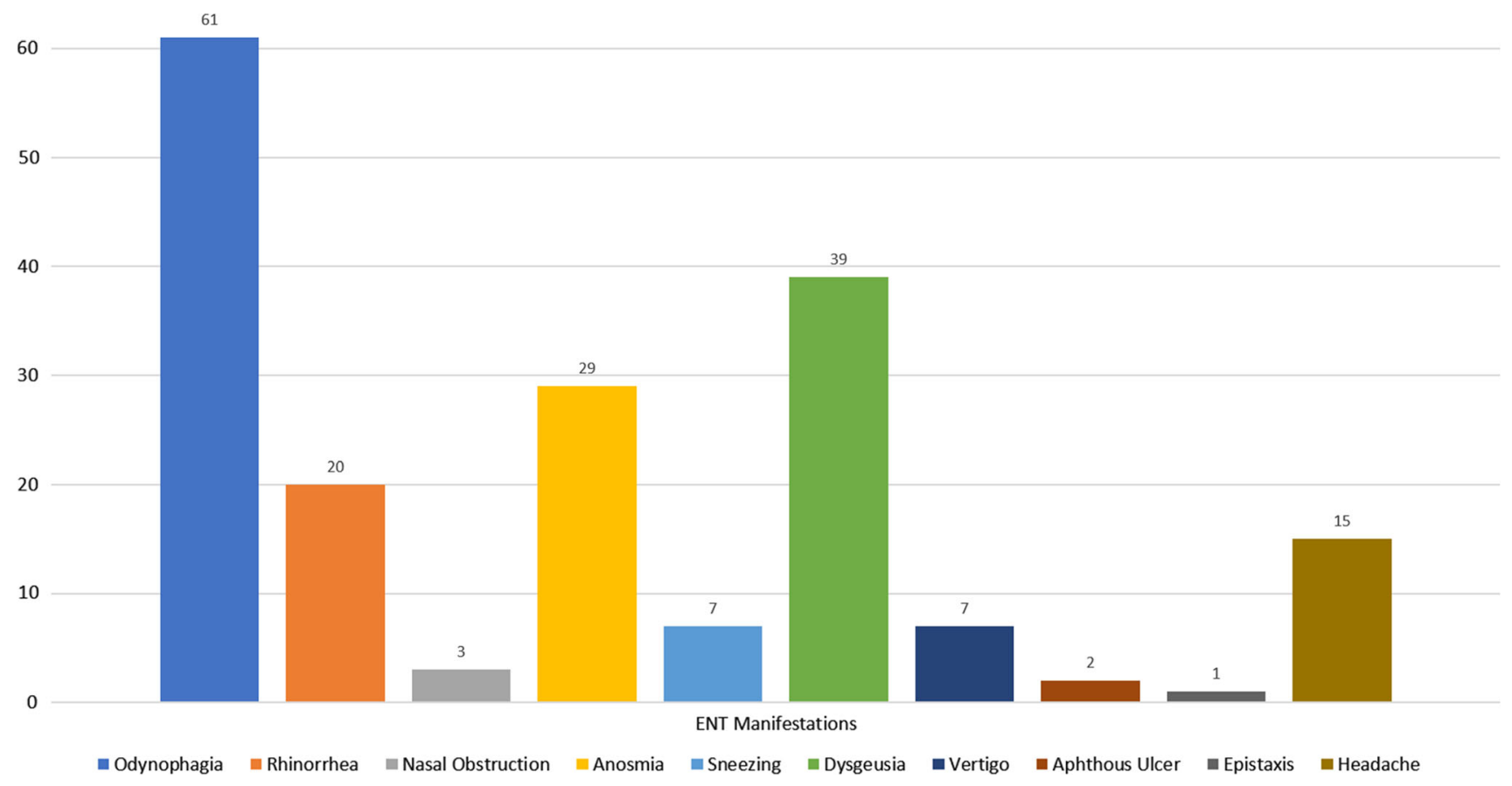

Fig. 2 Distribution of ENT manifestations

35

30

15

10

0
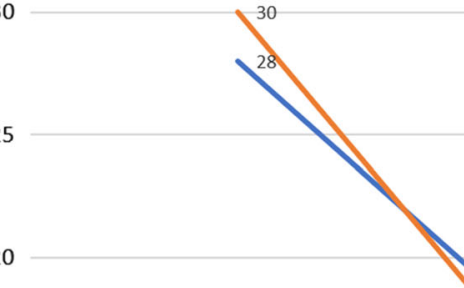

1 week

2 weeks

$$
\text { Anosmia Dysgeusia }
$$

Fig. 3 Temporal trends in the recovery pattern of anosmia and dysgeusia over a 4-week follow-up period

$73 \%$ in the questionnaire-based study of Kaye et al. and $79 \%$ reported by Hopkins et al. [8, 10]. A systematic review of 10 studies reporting anosmia and dysgeusia in
COVID-19, showed a pooled incidence of $52.73 \%$ for anosmia and $43.93 \%$ for dysgeusia [11]. Most of these studies are retrospective and questionnaire-based, making 
Table 2 Univariate analysis of predictors for anosmia/dysgeusia in COVID-19

\begin{tabular}{lll}
\hline & Relative risk & $p(95 \%$ CI $)$ \\
\hline Age & 1.04 & $0.66(0.76-1.18)$ \\
Sex (baseline male) & 0.95 & $0.64(0.41-1.72)$ \\
Comorbidity & 2.28 & $0.04(2.5-16.3)^{*}$ \\
Symptomatic vs asymptomatic & 0 & \\
NEWS 0 vs NEWS 1-2 & 0.73 & $0.37(0.38-1.4)$ \\
\hline
\end{tabular}

* indicate $p<0.05$, statistically significant

them prone to recall bias. This is evident from the study from Iran where UPSIT (University of Pennsylvania Smell Identification Test) was administered prospectively and 98\% were found to have different degrees of smell and taste disturbances [12]. The authors alluded to the fact that $35 \%$ of the patients were unaware of their smell and taste disturbance. These studies led the CDC to include "newonset smell and taste disturbance" to the list of early warning symptoms for COVID-19 [13]. Therefore, ENT symptoms, especially, smell and taste disturbances should be actively sought to avoid underreporting.

Unlike the studies quoted above, our study reveals only a $20 \%$ overall incidence of either anosmia or dysgeusia. This was a prospective study, therefore, under-reporting owing to recall bias was eliminated. There is a possibility of underestimating the true incidence in this study since a structured tool like UPSIT was not utilized. Nevertheless, our figures parallel the other studies conducted on Asian population (Mao et al. 5.1\% and Korean data-15.3\%) $[7,14]$. The explanation behind this ethnic disparity can be sought from the pathophysiology of SARS-Cov-2. SARSCov-2 gains entry into epithelial cells by binding to angiotensin-converting enzyme-2 protein (ACE-2) on the cell surface [15]. Though the olfactory neurons lack ACE2 , the virus may gain access through the sustentacular cells that express ACE-2, which facilitate entry into the olfactory fibres through exosomes [16]. This process along with the immunological response mounted by the damaged olfactory neurons form the basis for olfactory dysfunction [12]. The Asian population has a different allelic composition in ACE-2 and this polymorphism is responsible for the variable binding pattern of SARS-Cov-2 [17, 18].

COVID-19 associated smell and taste disturbance have certain salient features. Unlike non-COVID-19 post-viral anosmia, local factors causing "conductive" anosmia is not the underlying cause as seen by the limited number of patients manifesting other nasal symptoms as seen in this study $(\mathrm{n}=3)$ and the study by Mercante et al. (14\%) [8]. MRI focussing on the olfactory groove have identified hyperintensity on $\mathrm{T}-1$ weighted sequences due to underlying microbleeding and hemosiderin deposition [19]. This finding further provides credence to the "Neuro-COVID" hypothesis by Fotuhi et al. [20]. Autopsy studies have failed to demonstrate SARS-Cov-1 in olfactory neurons in the CNS [21] and the previously proven regenerative capacity of the olfactory bulb [22, 23], suggest that COVID-19 associated anosmia is unlikely to be related to a central cause. This is evinced by the excellent recovery rates reported in our study as well as other notable large series $[9,10]$. Previous studies have revealed that anosmia and dysgeusia are commonly associated with milder "nasal centric" COVID phenotype than the ones presenting with acute respiratory distress syndrome [10, 24]. This was the reason for basing our study entirely on the mild and asymptomatic population. This demographic subgroup primarily consists of younger individuals without comorbid illnesses. Identification of this subset of patients is paramount from an epidemiologic point of view as they have been identified as having a high level of virus shedding, thereby, propagating the disease in the community [25]. Since anosmia has been identified as a marker for COVID19, these ENT manifestations should be actively sought, especially in mild or asymptomatic individuals.

This was purely an observational study on a prospective cohort. No patient received any active intervention directed towards treatment of anosmia and dysgeusia. There are anecdotal reports highlighting the effectiveness of systemic and nasal steroid and olfactory retraining [26, 27]. These need to be validated in randomized controlled trials.

\section{Conclusion}

This prospective series highlights the importance of screening otolaryngologic manifestations in COVID-19. Smell and taste disturbance can predate other characteristic COVID-19 symptoms and thus mandate suspect isolation and testing. Though of considerable concern to the emotional well-being and quality of life of a patient, the excellent recovery rates demonstrated in this report, will be valuable in patient counselling and follow-up.

\section{Acknowledgements None.}

Author's Contribution Data collection: SP, AM, AK, PS, BS, LD, CK, RG. Analysis: SP, AK, KS, AT, SB, AM, VPM, PT, SV. Writing of the manuscript: SP, KS, AT.

\section{Funding None.}

Availability of Data and Materials The complete data pertaining to this study can be made available by the corresponding author if required for individual patient data metaanalysis. 


\section{Compliance with Ethical Standards}

\section{Conflict of interest None.}

Ethical Approval This study was a part of two ongoing clinical trials for which Institutional Ethical Clearance was obtained: IEC-250/ 17.04.2020 and IEC-251/17.04.2020.

Consent to Participate Informed written consent was obtained from all the participants prior to the study.

Consent for Publication Information about publishing the results in peer-reviewed journals was mentioned in detail to all the participants. All measures were undertaken to ensure anonymity of an individual during the entire process.

\section{References}

1. Coronavirus Update (Live): $21,617,697$ cases and 768,995 deaths from COVID-19 virus pandemic-Worldometer [Internet]. [cited 2020 Aug 16]. https://www.worldometers.info/coronavirus/

2. Gao Z, Xu Y, Sun C, Xang X, Guo Y, Qiu S et al (2020) A systematic review of asymptomatic infections with COVID-19. J Microbiol Immunol Infect. https://doi.org/10.1016/ j.jmii.2020.05.001

3. Zhu N, Li C, Ning SS, Chen S, Cao L, Yang GJ et al (2020) Epidemiological characteristics of COVID-19 in Shaanxi province. Zhonghua Liu Xing Bing Xue Za Zhi Zhonghua Liuxingbingxue Zazhi 41:E034

4. Krajewska J, Krajewski W, Zub K, Zatoński T (2020) COVID-19 in otolaryngologist practice: a review of current knowledge. Eur Arch Oto-Rhino-Laryngol 277:1885-1897

5. El-Anwar MW, Elzayat S, Fouad YA (2020) ENT manifestation in COVID-19 patients. Auris Nasus Larynx 47(4):559-564

6. Lovato A, de Filippis C (2020) Clinical presentation of COVID19: a systematic review focusing on upper airway symptoms. Ear Nose Throat J. https://doi.org/10.1177/0145561320920762

7. Mao L, Jin H, Wang M, Hu Y, Chen S, He Q et al (2020) Neurologic manifestations of hospitalized patients with coronavirus disease 2019 in Wuhan, China. JAMA Neurol 77(6):683-690

8. Mercante G, Ferreli F, De Virgilio A, Gaino F, Bari DM, Colombo G et al (2020) Prevalence of taste and smell dysfunction in coronavirus disease 2019. JAMA Otolaryngol Head Neck Surg 146:723-728

9. Kaye R, Chang CWD, Kazahaya K, Brereton J, Denneny JC (2020) COVID-19 anosmia reporting tool: initial findings. Otolaryngol Head Neck Surg 163:132-134

10. Hopkins C, Surda P, Whitehead E, Kumar BN (2020) Early recovery following new onset anosmia during the COVID-19 pandemic - an observational cohort study. J Otolaryngol Head Neck Surg 49:26

11. Tong JY, Wong A, Zhu D, Fastenberg JH, Tham T (2020) The prevalence of olfactory and gustatory dysfunction in COVID-19 patients: a systematic review and meta-analysis. Otolaryngol Head Neck Surg 163:3-11
12. Moein ST, Hashemian SM, Mansourafshar B, Khorram-Tousi A, Tabarsi P, Doty RL (2020) Smell dysfunction: a biomarker for COVID-19. Int Forum Allergy Rhinol 10:944-950

13. CDC. Coronavirus Disease 2019 (COVID-19)—Symptoms [Internet]. Centers for Disease Control and Prevention. 2020 [cited 2020 Aug 17]. https://www.cdc.gov/coronavirus/2019ncov/symptoms-testing/symptoms.html

14. $15 \%$ of COVID-19 patients lose sense of smell or taste: data [Internet]. [cited 2020 Aug 17]. https://www.koreatimes. co.kr/www/nation/2020/03/119_286790.html

15. Qi F, Qian S, Zhang S, Zhang Z (2020) Single cell RNA sequencing of 13 human tissues identify cell types and receptors of human coronaviruses. Biochem Biophys Res Commun 526:135-140

16. Butowt R, Bilinska K (2020) SARS-CoV-2: olfaction, brain infection, and the urgent need for clinical samples allowing earlier virus detection. ACS Chem Neurosci 11:1200-1203

17. Cao Y, Li L, Feng Z, Wan S, Huang P, Sun X et al (2020) Comparative genetic analysis of the novel coronavirus (2019nCoV/SARS-CoV-2) receptor ACE2 in different populations. Cell Discov 6:11

18. Li W, Zhang C, Sui J, Kuhn JH, Moore MJ, Luo S et al (2005) Receptor and viral determinants of SARS-coronavirus adaptation to human ACE2. EMBO J 24:1634-1643

19. Aragão MFVV, Leal MC, Cartaxo Filho OQ, Fonseca TM, Valença MM (2020) Anosmia in COVID-19 associated with injury to the olfactory bulbs evident on MRI. AJNR Am J Neuroradiol 41(9):1703-1706

20. Fotuhi M, Mian A, Meysami S, Raji CA (2020) Neurobiology of COVID-19. J Alzheimers Dis JAD 76:3-19

21. Gu J, Gong E, Zhang B, Zheng J, Gao Z, Zhong Y et al (2005) Multiple organ infection and the pathogenesis of SARS. J Exp Med 202:415-424

22. Chang SY, Glezer I (2018) The balance between efficient antiinflammatory treatment and neuronal regeneration in the olfactory epithelium. Neural Regen Res 13:1711-1714

23. Joiner AM, Green WW, McIntyre JC, Allen BL, Schwob JE, Martens JR (2015) Primary cilia on horizontal basal cells regulate regeneration of the olfactory epithelium. J Neurosci 35:13761-13772

24. Giacomelli A, Pezzati L, Conti F, Bernacchia D, Siano M, Oreni L et al (2020) Self-reported olfactory and taste disorders in patients with severe acute respiratory coronavirus 2 infection: a cross-sectional study. Clin Infect Dis 71:889-890

25. Wölfel R, Corman VM, Guggemos W, Seilmaier M, Zange S, Müller MA et al (2020) Virological assessment of hospitalized patients with COVID-2019. Nature 581:465-469

26. Loss of sense of smell as marker of COVID.pdf [Internet]. [cited 2020 Aug 18]. https://www.entuk.org/sites/default/ files/files/Loss $\% 20$ of $\% 20$ sense $\% 20$ of $\% 20$ smell $\% 20$ as $\%$ 20marker\%20of\%20COVID.pdf

27. Sorokowska A, Drechsler E, Karwowski M, Hummel T (2017) Effects of olfactory training: a meta-analysis. Rhinology $55: 17-26$

Publisher's Note Springer Nature remains neutral with regard to jurisdictional claims in published maps and institutional affiliations. 